\title{
OPEN Dynamically tunable switch and filter in single slot cavity structure
}

\author{
Boxun $\mathrm{Li}^{1}$, Lili Zeng ${ }^{2}$, Xingjiao Zhang ${ }^{1}$, Biao He ${ }^{3}$, Kun Liao ${ }^{1}$, Kun Liu ${ }^{1}$ \& Bin Wang ${ }^{1}$ \\ A single slot cavity coupled with two waveguides has been researched in theory and simulation. The \\ results comparison between theory and simulation shows they agree well. It is found that the lateral \\ displacement $S$ plays an important role in transmission properties. Moreover, increasing the width \\ of the slot cavity results in the emergence of new resonant peaks. At the same time, the shift of the \\ resonant peaks have been explained well. The slot cavity with Kerr nonlinear material can act as a \\ dynamically tunable four channel switch and filter. The single slot cavity has the advantages of simple \\ and compact structure, easy fabrication, and the excellent properties are helpful to control light in \\ photonics circuits.
}

Received: 21 December 2018
Accepted: 23 September 2019 Published online: 10 October 2019
Surface plasmons (SPs) are electromagnetic waves coupled to free electron oscillations traveling along the metal-dielectric interface. Due to the capabilities of overcoming diffraction limit and confining light in deep sub-wavelength dimension ${ }^{1}$. SPs provides possibility for nanodevices with high miniaturization and integration ${ }^{2}$. Consequently, the SPs is considered as a promising way for realization of highly integrated optical circuits ${ }^{3-8}$.

The metal-dielectric-metal (MDM) waveguide structure, with the remarkable advantages of long propagation distance, easy fabrication and convient integration, has attracted tremendous attentions $\mathrm{s}^{9,10}$. Some researchers had investigated the tunable plasmonic structures based on MDM waveguide. It can also be applied in sensor and filter ${ }^{11-17}$. For example, Xu et al. studied the effect of nano defect in stub on transmission properties ${ }^{13}$. Zhan et al. researched Fano resonance in MDM waveguide which can be acted as a tunable nanoplasmonic sensor ${ }^{14}$. Li et al. reported Fano resonance based on multimode and degenerate mode interference ${ }^{16}$. Huang et al. reported the sensing performance of the MDM waveguide which is based on transparency effect can be enhanced ${ }^{17}$. For dynamically tunable with different structures, the traditional realization method is to directly adjust the parameters. However, to a given structure, once the plasmonic nanostructure has been fabricated, it is unrealistic to achieve adjustable the properties by changing the parameters. More efforts still need to be made to realize the dynamic adjustable devices.

In this paper, a single slot cavity has been investigated theoretically and numerically. The simulated results agree well with the the theoretical results. The lateral displacement $S$ plays an important role in the transmission property. The first and second order mode can be separated without interference by choosing an appropriate $S$. Attributing to the superposition of resonant modes, it is found that new resonant peaks emerge by increasing the width of slot cavity. The shift of the resonant peaks have been explained well. The results reveal that the single slot cavity with Kerr nonlinear material can act as a dynamically tunable four channel switch and filter. This work paves a new way towards the realization of dynamic control of light in photonics circuits.

\section{Model and Method}

The proposed single slot cavity waveguide coupling structure is schematically shown in Fig. 1, which consists of a slot cavity coupled with two waveguides, and the single slot cavity waveguide coupling structure is a 2-D metallic structure which is infinitely long in $\mathrm{z}$-axis. The incident light is along the $\mathrm{x}$ direction with TM polarization (the magnetic field is parallel to $\mathrm{z}$ direction). TM-polarized wave is emitted from $P$ and propagates to $Q$. $P_{\mathrm{P}}$ and $P_{Q}$ stand for input and output power flows, respectively. The transmission is defined as $T=P_{\mathrm{Q}} / P_{\mathrm{P}}$. The width of waveguides and slot cavity are set to be $w_{1}$ and $w$, where the width of waveguides $w_{1}$ equal to $50 \mathrm{~nm}$. $L$ stands for the length of slot cavity. $S_{1}$ and $S_{2}$ present the lateral displacement between the central of two waveguides and reference plane of slot cavity. The distance between slot cavity and two waveguides is $g$, which is a constant. Here, the orange and white areas are silver and dielectric, respectively. Unless otherwise specified, the dielectric is air in this manuscript. The Drude model $\varepsilon_{m}(\omega)=\varepsilon_{\infty}-\omega_{p} /\left(\omega^{2}+i \omega \gamma p\right)$ is introduced to characterize

${ }^{1}$ School of Mechatronics Engineering, Ping Xiang University, Ping Xiang, 337055, P.R. China. ${ }^{2}$ College of Solar Energy Engineering, Hunan Vocational Institute of Technology, Xiangtan, 411104, P.R. China. ${ }^{3}$ College of Physics and Electronics, Central South University, Changsha, 410083, P.R. China. Correspondence and requests for materials should be addressed to L.Z. (email: 405515532@qq.com) orX.Z. (email: zhangxingjiao521@163.com) 


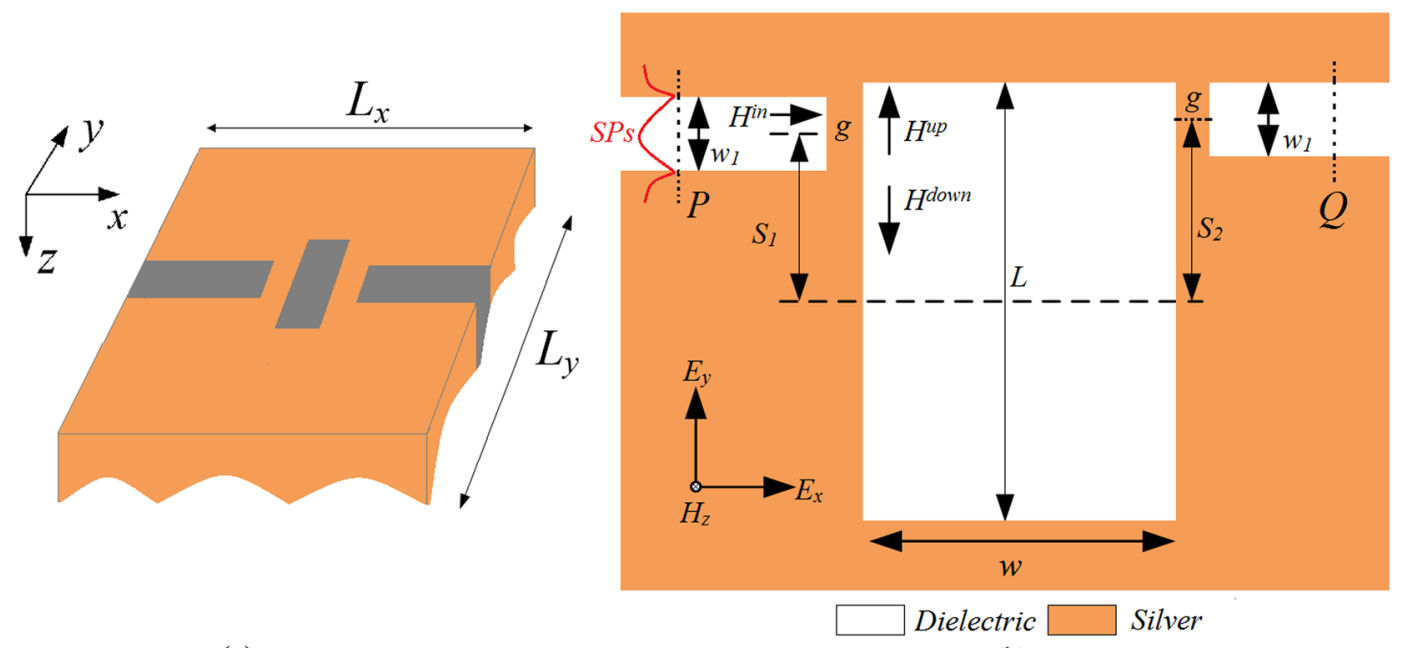

(a)

(b)

Figure 1. The (a)schematic and (b)top view of the 2D single slot cavity waveguide coupling structure.
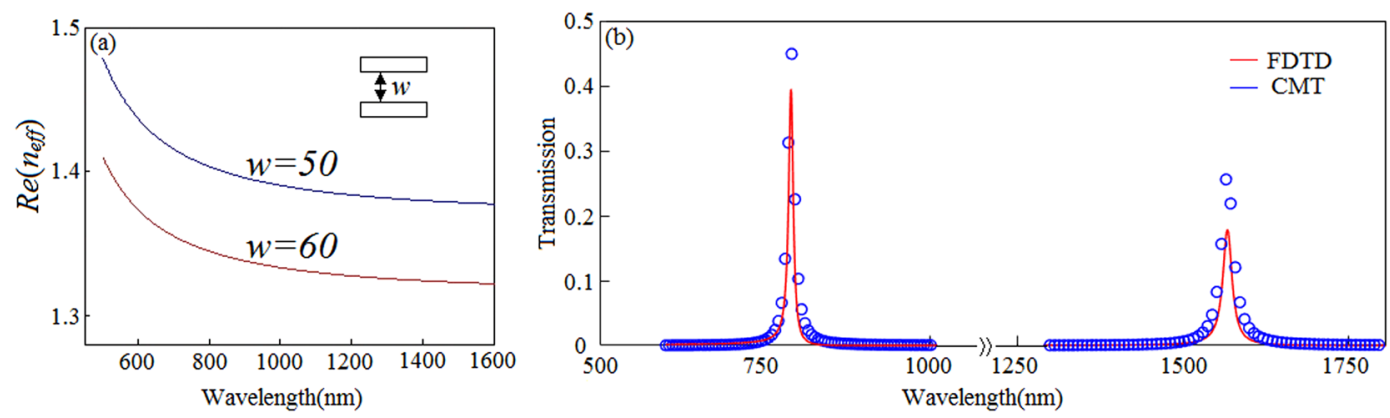

Figure 2. (a) Effective refractive index as function of wavelength with various $w(\mathrm{~nm})$. (b) Simulated (red line) and theoretical results (blue cycle) with $S_{1}=S_{2}=275 \mathrm{~nm}, L=600 \mathrm{~nm}$ and $w=50 \mathrm{~nm}$.

the complex relative permittivity of silver, where the dielectric constant $\varepsilon_{\infty}=3.7$ at the infinite frequency, the bulk plasma frequency $\omega_{p}=1.38 \times 10^{16} \mathrm{rad} / \mathrm{s}, \omega$ stands for the angle frequency of incident wave, the damping rate $\gamma_{p}=2.73 \times 10^{13} \mathrm{rad} / \mathrm{s}$ characters the absorption $\operatorname{loss}^{18}$. The finite-difference time-domain (FDTD) with perfectly matched layer (PML) absorbing boundary is introduced to investigate the configuration. The computational space is $L_{x} \times L_{y}=1000 \mathrm{~nm} \times 1000 \mathrm{~nm}$, the spatial and temporal steps are set as $\Delta x=\Delta y=5 \mathrm{~nm}$, and $\Delta t=\Delta x / 2 c$ (c is the velocity of light in vacuum), respectively, which are sufficient for the convergence of numerical results.

\section{Results and Discussions}

When SPs are coupled into the slot cavity, we define $\varphi$ to be the phase delay per round-trip in slot cavity, and $\varphi$ has the relation ${ }^{19} \varphi=4 \pi n_{e f f} L / \lambda_{m}+\varphi_{r}$, where $n_{e f f}, \lambda_{m}$ and $\varphi_{r}$ are the effective refractive index, resonance wavelength and phase shift of a beam reflected on the upper and lower facets of slot cavity, respectively. The $\varphi_{r}$ is very little which has no obvious effect on the resonant wavelength. And the effective refractive index can be obtained from the dispersion equation ${ }^{20-22}$ :

$$
\varepsilon_{m} \sqrt{n_{e f f}^{2}-\varepsilon_{d}} \tanh \left(\frac{w \pi \sqrt{n_{e f f}^{2}-\varepsilon_{d}}}{\lambda}\right)+\varepsilon_{d} \sqrt{n_{e f f}^{2}-\varepsilon_{m}}=0
$$

Where $\varepsilon_{m}$ and $\varepsilon_{d}$ stand for the dielectric constant of metal and dielectric waveguide with a width of $w$, respectively. Figure 2(a) shows $n_{\text {eff }}$ as a function of wavelength with various $w$. It is found that the value has significant various in the visible range. With the increasing of wavelength, the curve becomes relatively flatter when the wavelength increases from $800 \mathrm{~nm}$ to $1600 \mathrm{~nm}$. Moreover, it is demonstrated from Fig. 2(a) that the wider the width and the smaller the value of $n_{\text {eff }}$ at a given wavelength.

For the single cavity waveguide coupling structure, a temporal coupled mode theory (CMT) is introduced to research the transmission features. In order to simplify the calculation, we neglect the propagation and coupling losses as well as the phase shift, the temporal normalized mode amplitude $a$ of slot cavity can be expressed as ${ }^{23}$ 


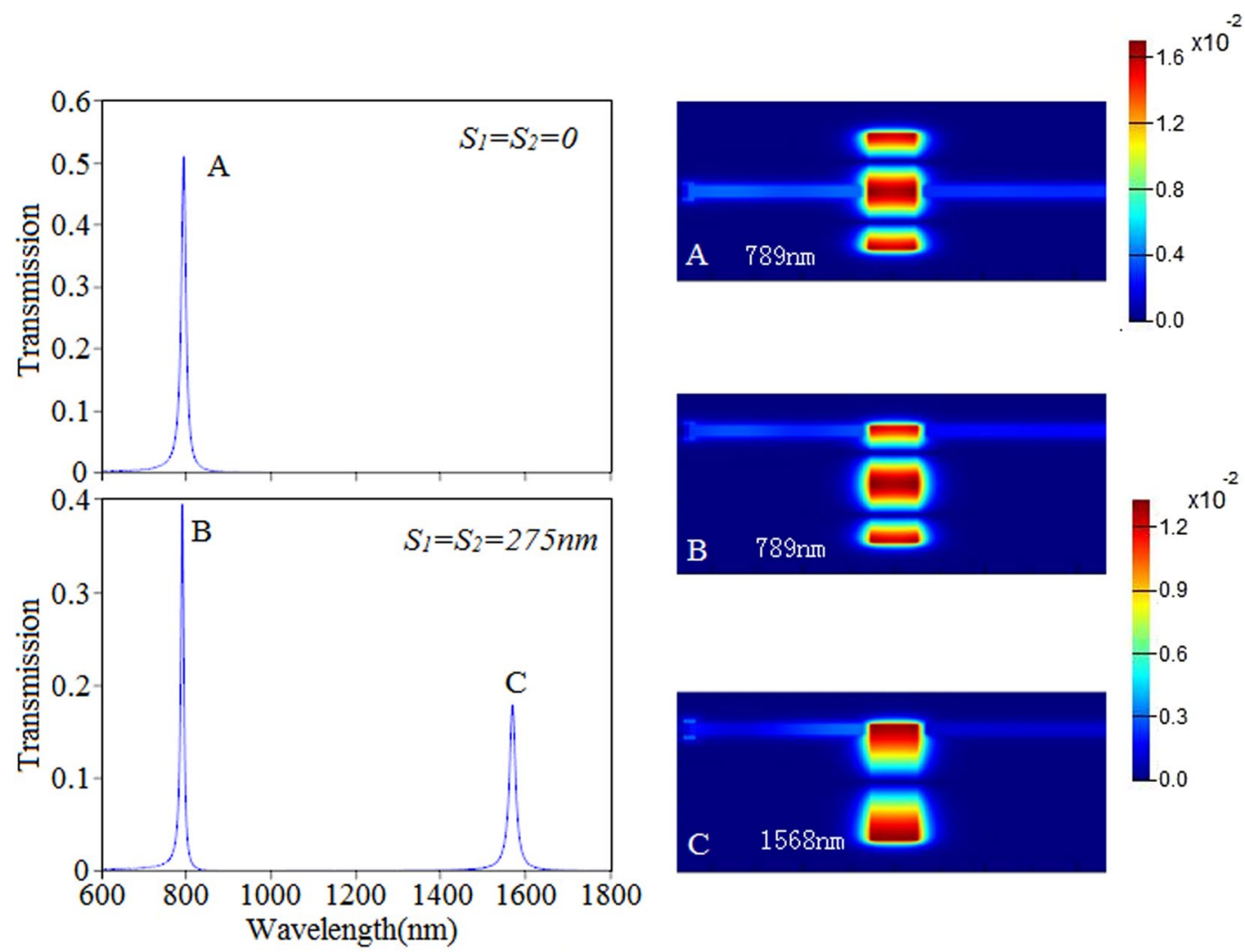

(a)

(b)

Figure 3. (a) The transmission spectral with different $S_{1}$ and $S_{2}$. (b) The magnetic fields of three peaks A, B and C.

$$
\frac{d a}{d t}=\left(-j \omega_{0}-k_{0}-k_{1}-k_{2}\right) a+A_{+1} \sqrt{k_{1}}+A_{+2} \sqrt{k_{2}}
$$

Where $\omega_{0}$ represents the resonance frequency of the slot cavity, $k_{0}, k_{1}$ and $k_{2}$ respectively stand for the internal loss of slot cavity and the coupling loss to two waveguides, which satisfy the relationships $k_{i}=\omega_{0} / 2 Q_{i}(i=0,1,2)$. Here $Q_{i}$ are the related quality factors. $Q_{1}$ and $Q_{2}$ are controlled by the coupling distances $\mathrm{g}$, which has been proved in some reference ${ }^{24}$. And there is a contradiction between quality factor and transmittance. That is the transmittance decreases and the quality factor increases with the increasing of distance $g$. According to the energy conservation, the transmission $T$ of the single cavity waveguide coupling structure can be derived as ${ }^{25}$

$$
T=\left|\frac{\sqrt{2 k_{1} k_{2}}}{j\left(\omega-\omega_{0}\right)+k_{0}+k_{1}+k_{2}}\right|^{2}
$$

Figure 2(b) describes the simulated and theoretical results which are presented by red line and blue cycle, respectively. Most obviously, the simulated results agree well with theoretical results. A little deviation can be attributed to the assumptions of neglecting coupling and propagation losses as well as phase shift.

Figure 3(a) shows the transmission spectral with various distance between the centre of two waveguides and reference plane of slot cavity. When $S_{1}=S_{2}=0$, it is found that there is a transmission peak A which locates at $789 \mathrm{~nm}$. However, when $S_{1}=S_{2}=275 \mathrm{~nm}$, there are two transmission peaks B and C which are located at $789 \mathrm{~nm}$ and $1568 \mathrm{~nm}$, respectively. What needs to be pointed out is that the transmittance of peak $\mathrm{A}$ is about $52 \%$ which is higher than that of peak B with a transmittance $39 \%$. In order to investigate the abnormal phenomenon, we have plot the magnetic fields of peak A, B and C, which are illustrated in Fig. 3(b). Obviously, the distributions of fields of A and B are similar. And the intensity of A is stronger than that of B. It is concluded from magnetic fields of peak A that the stable standing waves has been excited in the slot cavity when the resonant condition is satisfied, and the peak A is the second order mode of the slot cavity. Similarly, the magnetic fields of B and C correspond to the second and the first order mode, respectively. According to magnetic fields, due to coupled resonance between slot cavity and waveguide, it is concluded that the energy can indirectly couple into output waveguide when the output waveguide locates at the high energy areas of the slot cavity. It is obvious that the first order mode do not exist in the slot cavity when $S_{1}=S_{2}=0$, while the first order and the second order mode can exist in the slot cavity when $S_{1}=S_{2}=275 \mathrm{~nm}$. Based on the results above, we can derive that the second order mode can couple into output waveguide while the first order mode cannot when $S_{1}=275 \mathrm{~nm}$ and $S_{2}=0$. 


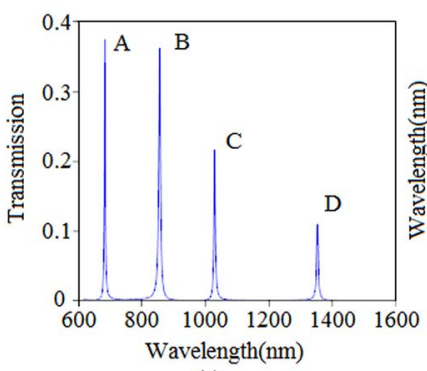

(a)

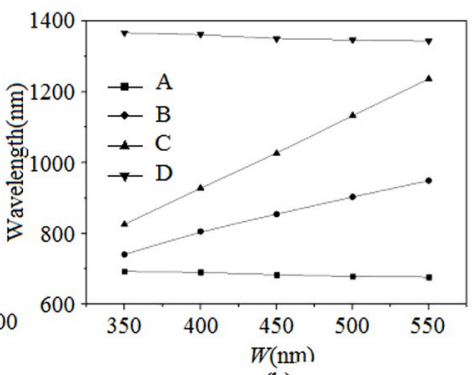

(b)

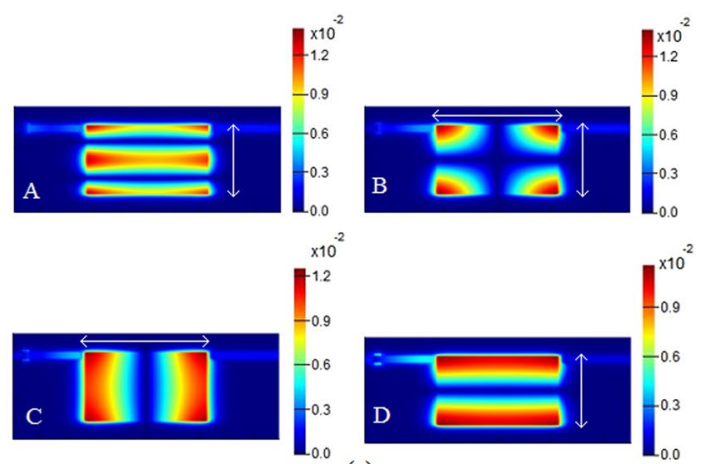

(c)

Figure 4. (a) The transmission spectrum with $w=450 \mathrm{~nm}$, where other parameters are $S_{1}=S_{2}=275 \mathrm{~nm}$ and $L=600 \mathrm{~nm}$. (b) The wavelengths of A, B, C and D with different width $w$. (c) The magnetic fields of A, B, C and $\mathrm{D}$ in $(\mathbf{a})$.

Based on the superposition principle of optics ${ }^{26}$, we qualitatively analyze the transmission spectral with different $S_{1}$ and $S_{2}$. When the resonance condition is satisfied ${ }^{19}: \Phi=\beta_{m} \cdot 2 L+\theta=2 m \pi$, where $\theta$ stands for the phase shift of a beam reflected on the up and down facets of the slot cavity. $\beta_{m}$ is the propagation constant of SPs corresponding to the $m^{\text {st }}$ order resonance mode of the slot cavity. Just as shown in Fig. 1, the input field $H^{\text {in }}$ inside the slot cavity is divided into two parts, the $H^{u p}$ and $H^{\text {down }}$, which have the relation of $H^{u p}=H^{\text {down }}=H^{i n} / 2=H_{0}$ and propagate in opposite directions. Intensity inside the slot cavity with an arbitrary input position $S$ can be expressed as:

$$
\begin{aligned}
H_{m}(x, t)= & \frac{2 H_{0} \cos \left(\beta_{m} x-0.5 \beta_{m} L\right)}{\sigma} \times\left\{\exp \left[j\left(1.5 \beta_{m} L-\beta_{m} S_{1}\right)\right]+\exp \left[j\left(\beta_{m} S_{1}+0.5 \beta_{m} L\right)\right]\right\} \\
& \times \exp \left(-j \omega_{m} t\right)
\end{aligned}
$$

Where $\sigma$ stands for the dissipation of the light propagating. Neglect the phase shift $\theta$, one has $\Phi=\beta_{m} \cdot 2 L=2 m \pi$, for the first order mode $(m=1)$, the Eq. (3) can be expressed as

$$
H_{1}(x, t)=2 H_{0} \cos \left(\beta_{1} x-0.5 \pi\right) \times\left[-2 \sin \left(\beta_{1} S_{1}\right)\right] \times \exp \left(-j \omega_{1} t\right) / \sigma
$$

For the second order mode ( $m=2)$, the Eq. (3) can be written as:

$$
H_{2}(x, t)=2 H_{0} \cos \left(\beta_{2} x-\pi\right) \times\left[-2 \cos \left(\beta_{2} S\right)\right] \times \exp \left(-j \omega_{2} t\right) / \sigma
$$

According to the equations above, it is concluded that when $S_{1}=0, H_{1}=0, H_{2} \neq 0$, that is to say, the first order mode cannot exist in the slot cavity, while the second order mode can exist when $S_{1}=0$. At the same time, when $S_{1}=275 \mathrm{~nm}$, the first and the second order mode can exist in the slot cavity. The equation helps explain why different displacement results in different transmission property. What is worth mentioning is that the transmission spectral of the single cavity waveguide coupling structure has a high quality factor (QF), where the QF of peak B and $\mathrm{C}$ are 94 and 98, respectively. These excellent characters can be applied in filter.

In order to investigate the effect of parameters on transmission spectrum, we change the width of the slot cavity, it is found that four peaks appear in the spectrum when the width increases to $350 \mathrm{~nm}$. Obviously, a phenomenon with two new peaks emerges in the Fig. 4(a). As is shown in Fig. 4(b), it is seen that the peaks B and C have a obvious red shift with the increasing of width $w$, while the peaks $\mathrm{A}$ and $\mathrm{D}$ have a opposite variation tendency. What is noteworthy is that the variation tendencies of peaks $\mathrm{B}$ and $\mathrm{C}$ are greater than that of $\mathrm{A}$ and $\mathrm{D}$. To explore the underlying mechanism, just as illustrated in Fig. 4(c), the magnetic fields of A, B, C and D in Fig. 4(a) are plot. Obviously, the fields of peaks A and D correspond to the second and the first order mode, respectively, which are similar to the Fig. 3(b). And the energy propagate along the vertical direction of the slot cavity which is shown by the white double arrow in Fig. 4(c). To the peak C, it is found that the energy propagate along the horizontal direction of the slot cavity. It is concluded from magnetic field of $B$ that the SPs propagate along the vertical and horizontal directions in the slot cavity, which can be deemed to the superposition of $\mathrm{C}$ and $\mathrm{D}$. To the peaks $\mathrm{A}$ and $\mathrm{D}$, due to the SPs propagate along vertical direction, the propagation length is $L$, and the width is $w$. According to the the phase delay equation $\left(\varphi=4 \pi n_{e f f} L / \lambda_{m}+\varphi_{r}\right)$ and dispersion Eq. (1), increasing the width $w$ means decreasing the $n_{e f f}$, which results in the decrease of resonant wavelength, and the transmission spectrum manifests a blue shift. Moreover, according to the Fig. 2(a), increasing width $w$ has not great impact on the $n_{\text {eff }}$, and the variation tendencies of peaks $\mathrm{A}$ and $\mathrm{D}$ change little. To the peak $\mathrm{C}$, the propagation length is $w$ and the width is $L$, increasing $w$ will increase the propagation length, and the resonant wavelength $\lambda_{m}$ will shift to long wavelength. With respect to the peak $\mathrm{B}$, the propagation length is $w+L^{27}$, the resonant wavelength also shift to long wavelength. In reference ${ }^{27}$, there is a similar conclusion to ours. To the four peaks A, B, C and D, they can be calculated quantificationally, due to the high QF, the properties can be also applied in filter.

In order to realize the function of dynamic regulation to the single slot cavity, the two waveguides and slot cavity are filled with a Kerr nonlinear material whose nonlinear refractive index can be adjustable with an external control (pumping) beam. Due to large third-order optical nonlinear susceptibility $\chi^{(3)}$ and ultra-fast response time which are important for switching function, $\mathrm{Au} / \mathrm{SiO}_{2}$ with $\chi^{(3)}=1.7 \times 10^{-7} \mathrm{esu}\left(2.37 \times 10^{-15} \mathrm{~m}^{2} / \mathrm{v}^{2}\right)^{28}$ is 


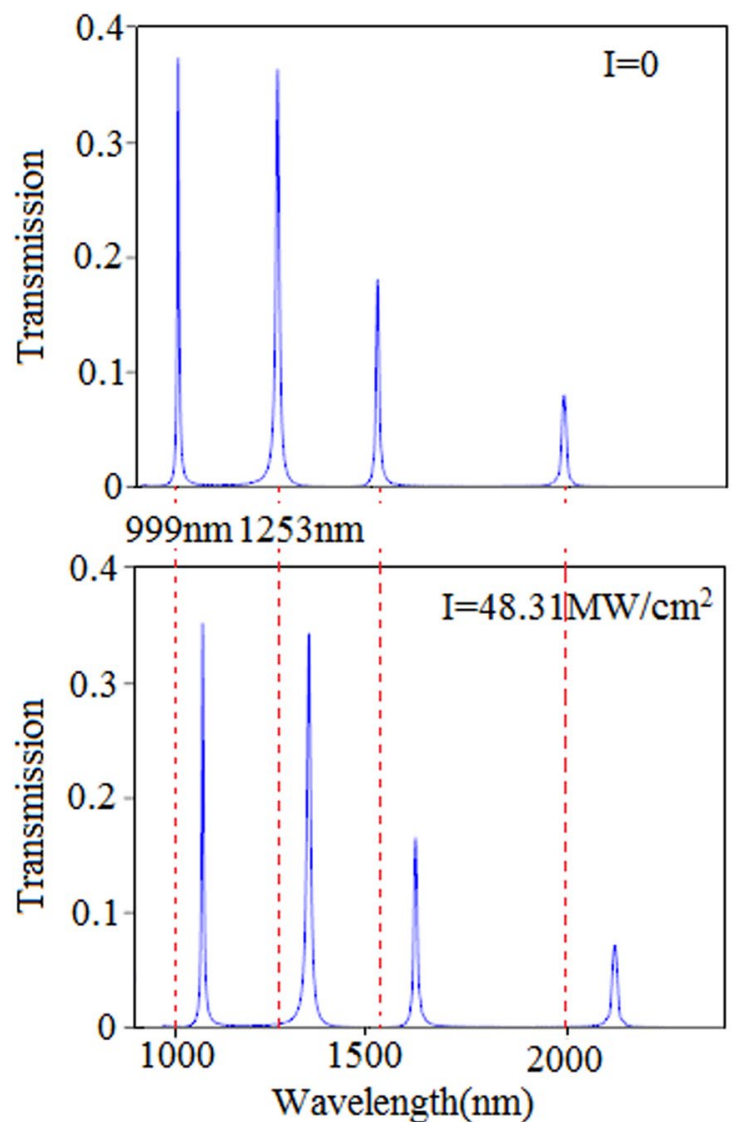

(a)
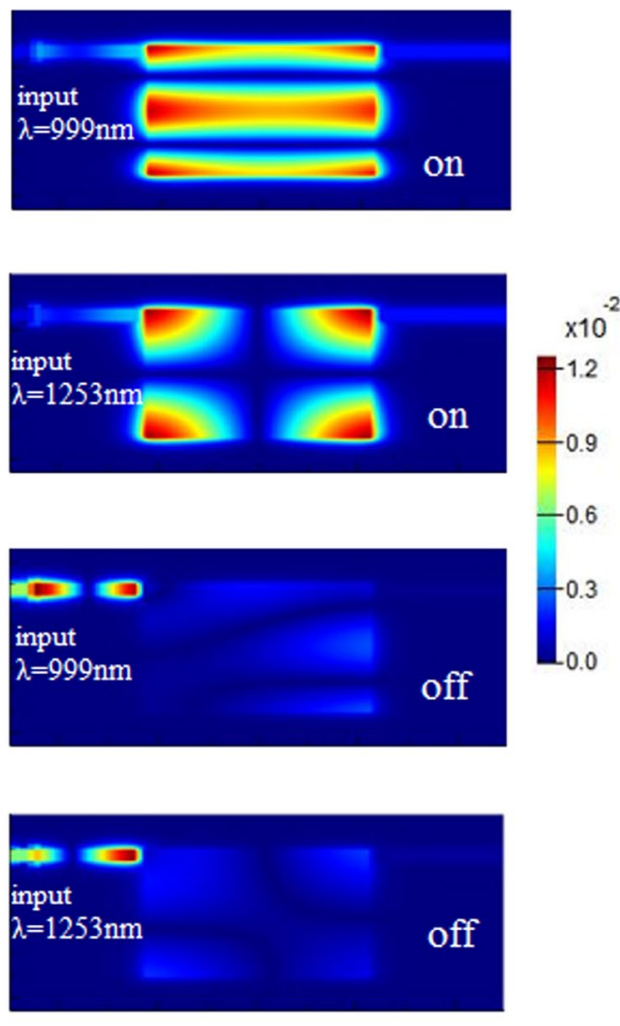

(b)

Figure 5. (a) The transmission spectra at two different pumping intensities with $L=600 \mathrm{~nm}, w=450 \mathrm{~nm}$. (b) The magnetic fields at wavelengths of $999 \mathrm{~nm}$ and $1253 \mathrm{~nm}$. The 'on' state without the pumping light and the 'off' state with the pumping light. The dielectric is Kerr nonlinear material.

\begin{tabular}{|c|c|c|c|c|}
\hline \multirow[b]{2}{*}{$\lambda(\mathbf{n m})$} & \multicolumn{2}{|l|}{$\mathrm{I}=\mathbf{0}$} & \multicolumn{2}{|c|}{$\mathrm{I}=48.31 \mathrm{MW} / \mathrm{cm}^{2}$} \\
\hline & Transmittance & State & Transmittance & State \\
\hline 999 & 0.37 & on & 0.009 & off \\
\hline 1066 & 0.004 & off & 0.35 & on \\
\hline 1253 & 0.36 & on & 0.007 & off \\
\hline 1340 & 0.007 & off & 0.35 & on \\
\hline 1517 & 0.18 & on & 0.008 & off \\
\hline 1610 & 0.003 & off & 0.17 & on \\
\hline 1989 & 0.12 & on & 0.005 & off \\
\hline 2115 & 0.006 & off & 0.1 & on \\
\hline
\end{tabular}

Table 1. The different wavelengths stay on/off state with and without pumping light.

chosen to fill in the slot cavity and two waveguides. The refractive index of is expressed as $n=n_{0}+n_{2} I^{29}$, where $n_{0}=1.47$ is the linear refractive index, $n_{2}=2.07 \times 10^{-9} \mathrm{~cm}^{2} / \mathrm{W}$ is the nonlinear refractive index coefficient and $I$ is the pumping beam intensity.

Figure 5(a) shows the transmission spectra at two different pumping intensities. It is found that the four peaks shift to long wavelength with changing the pumping intensity $I$. When the pumping intensity $I$ equals to 0 , the wavelength $999 \mathrm{~nm}$ with a transmittance of $37 \%$ can pass through the output port. With the pumping intensity $I$ increasing to $48.31 \mathrm{MW} / \mathrm{cm}^{2}$, the transmittance of wavelength $999 \mathrm{~nm}$ tends to 0 and cannot couple into output port. Likewise, the other three wavelengths have the similar variation tendency. Figure 5(b) displays the magnetic fields of $999 \mathrm{~nm}$ and $1253 \mathrm{~nm}$ without and with pumping light. It can be seen that the light can couple into output port without pumping light at the wavelengths of $999 \mathrm{~nm}$ and $1253 \mathrm{~nm}$. These results can be regarded as the 'on' state. Moreover, when pumping light with $I=48.31 \mathrm{MW} / \mathrm{cm}^{2}$ is introduced, the light cannot couple into the output port, which can be regarded as the 'off' state. Table 1 describes the different wavelengths stay 'on/off' state with and without pumping light. To a given wavelength, it is clear that the light can or cannot couple into 
output port when $I=0$ or $I=48.31 \mathrm{MW} / \mathrm{cm}^{2}$. In other words, the single slot cavity can be applied in dynamically tunable multi-switching by introducing pumping light. These excellent properties have an important application in optical circuits.

\section{Conclusion}

In sum, a single slot cavity has been investigated theoretically and numerically. The simulated results agree well with the theoretical results. The lateral displacement $S$ plays an important role in the transmission property. The first and second order mode can be separated without interference by choosing an appropriate $S$. Due to the superposition of resonant modes, it is found that new resonant peaks emerge by increasing the width of slot cavity. The shift of the resonant peaks have been explained well. The results reveal that the single slot cavity with Kerr nonlinear material can act as a dynamically tunable four channel switch and filter. The proposed single slot cavity has the advantages of simple and compact structure, easy fabrication, which paves a new way towards the realization of dynamic control of light in photonics circuits.

\section{References}

1. Gramotnev, D. \& Bozhevolnyi, S. Plasmonics beyond the diffraction limit. Nat. Photonics. 4(2), 83-91 (2010).

2. Ebbesen, T., Genet, C. \& Bozhevolnyi, S. Surface-plasmon circuitry. Phys. Today. 61(5), 44-50 (2008).

3. Fan, B. et al. Integrated sensor for ultra-thin layer sensing based on hybrid coupler with short-range surface plasmon polariton and dielectric waveguide. Appl. Phys. Lett. 102(6), 5186-3960 (2013).

4. Melikyan, A., Alloatti, L. \& Muslija, A. High-speed plasmonic phase modulators. Nat. Photonics. 8(8), 229-233 (2014).

5. Chen, J., Li, Z., Zhang, X., Xiao, J. \& Gong, Q. Submicron bidirectional all-optical plasmonic switches. Sci. Rep. 3(3), 1451 (2013).

6. Li, Z. et al. High performance broadband asymmetric polarization conversion due to polarization-dependent reflection. Plasmonics. 10(6), 1703-1711 (2015).

7. Zhang, C., Min, C., Du, L. \& Yuan, X. Perfect optical vortex enhanced surface plasmon excitation for plasmonic structured illumination microscopy imaging. Appl. Phys. Lett. 108(20), 780-782 (2016).

8. Ren, H., Li, X., Zhang, Q. \& Gu, M. On-chip noninterference angular momentum multiplexing of broadband light. Science 352(6287), 805-809 (2016).

9. Zeng, L., Li, B., Li, H. \& Wang, B. Nano sensing based on transparency effect in planar metamaterial. J. Mod. Opt. 64(12), 1158-1163 (2017).

10. He, Z., Li, H. \& Zhan, S. Oscillator Model Analysis for Slow Light in Bright-Dark-Dark Waveguide Systems. IEEE Photonic. Tech. L. 27(22), 2371-2374 (2015).

11. Chen, Z. et al. Tunable ultra-wide band-stop filter based on single-stub plasmonic-waveguide system. Appl. Phys. Express. 9(10), 102002 (2016).

12. Li, B. et al. The sensing applications in U-shape structure based on dual transparency windows. IEEE Photonic. J. 10(5), 5700808 (2018).

13. Xu, H. et al. Influential and theoretical analysis of nano-defect in the stub resonator. Sci. Rep. 6, 30877 (2016).

14. Zhan, S. et al. Tunable nanoplasmonic sensor based on the asymmetric degree of Fano resonance in MDM waveguide. Sci. Rep. 6 , 22428 (2016).

15. Li, B. et al. Sensing application in Fano resonance with T-shape structure. J. Light. Tech. 34(14), 3342-3347 (2016).

16. Li, S. et al. Fano resonances based on multimode and degenerate mode interference in plasmonic resonator system. Opt. Express. 25(4), 3525-3533 (2017).

17. Huang, Y., Min, C., Dastmalchi, P. \& Veronis, G. Slow-light enhanced subwavelength plasmonic waveguide refractive index sensors. Opt. Express. 23(11), 14922-14936 (2015).

18. Palik, E. D. Handbook of Optical Constants of Solids. Academic Press (1998).

19. Li, B. et al. Theoretical analysis and applications in inverse t-shape structure. J. Opt. Soc. Am. A. 33(5), 811 (2016).

20. Park, J., Kim, H. \& Lee, B. High order plasmonic bragg reflection in the metal-insulator-metal waveguide bragg grating. Opt. Express. 16(1), $413(2008)$.

21. Han, Z., Forsberg, E. \& He, S. Surface plasmon bragg gratings formed in metal-insulator-metal waveguides. IEEE Photonic. Tech. Lett. 19(2), 91-93 (2007).

22. Johnson, P. \& Christy, R. Optical constants of the noble metals. Phys. Rev. B. 6(12), 4370-4379 (1972).

23. Li, Q., Wang, T., Su, Y., Yan, M. \& Qiu, M. Coupled mode theory analysis of mode-splitting in coupled cavity system. Opt. Express. 18(8), 8367 (2010).

24. Wen, K., Hu, Y. \& Chen, L. Fano resonance based on end-coupled cascaded-ring MIM waveguides structure. Plasmonics 12(6), 1875-1880 (2017).

25. Lu, H., Liu, X., Mao, D., Wang, L. \& Gong, Y. Tunable band-pass plasmonic waveguide filters with nanodisk resonators. Opt. Express. 18(17), $17922(2010)$.

26. Akhmanov, S. \& Nikitin, S. Physical Optics (Oxford University Press, 1997).

27. Chen, Z. \& Yu, L. Multiple Fano Resonances Based on Different Waveguide Modes in a Symmetry Breaking Plasmonic System. IEEE Photonic. J. 6(6), 1-8 (2014).

28. Wong, G. et al. Origin of third-order optical nonlinearity in au:sio2 composite films on femtosecond and picosecond time scales. Opt. Lett. 23(5), 388 (1998).

29. Tao, J., Wang, Q. \& Huang, X. All-optical plasmonic switches based on coupled nano-disk cavity structures containing nonlinear material. Plasmonics 6(4), 753 (2011).

\section{Acknowledgements}

This work was funded by the Research Fund for the National Natural Science Foundation of China (Grant number. 11747020), the Natural science foundation of Hunan province (grant number 2019JJ70060), the Science and technology project of Hunan provincial education department (grant number 18C1444), the Science and technology project of Jiangxi provincial education department (grant number GJJ171131, GJJ161264) and the 2017 Science and technology bureau project of Pingxiang municipal.

\section{Author Contributions}

This research was planned by Boxun Li and Lili Zeng. Boxun Li developed the analytic theory. Numberical simulation was performed by Boxun Li, Lili Zeng, Xingjiao Zhang and Kun Liao. The authors Boxun Li, Lili Zeng, Xingjiao Zhang, Biao He, Kun Liao, Kun Liu and Bin Wang discussed the results. Boxun Li wrote the manuscript. 


\section{Additional Information}

Competing Interests: The authors declare no competing interests.

Publisher's note Springer Nature remains neutral with regard to jurisdictional claims in published maps and institutional affiliations.

(c) Open Access This article is licensed under a Creative Commons Attribution 4.0 International License, which permits use, sharing, adaptation, distribution and reproduction in any medium or format, as long as you give appropriate credit to the original author(s) and the source, provide a link to the Creative Commons license, and indicate if changes were made. The images or other third party material in this article are included in the article's Creative Commons license, unless indicated otherwise in a credit line to the material. If material is not included in the article's Creative Commons license and your intended use is not permitted by statutory regulation or exceeds the permitted use, you will need to obtain permission directly from the copyright holder. To view a copy of this license, visit http://creativecommons.org/licenses/by/4.0/.

(C) The Author(s) 2019 